\title{
Effects of three-body collisions in a two-mode Bose-Einstein condensate
}

Cite as: J. Math. Phys. 56, 112102 (2015); https://doi.org/10.1063/1.4936314

Submitted: 26 August 2015 . Accepted: 10 November 2015 . Published Online: 25

November 2015

Carlos Sabín, Pablo Barberis-Blostein, Cristopher Hernández, Robert B. Mann, and Ivette Fuentes

\section{ARTICLES YOU MAY BE INTERESTED IN}

Quantum mechanical derivation of the Wallis formula for $\pi$ Journal of Mathematical Physics 56, 112101 (2015); https:// doi.org/10.1063/1.4930800

An introduction to Pound-Drever-Hall laser frequency stabilization

American Journal of Physics 69, 79 (2001); https://doi.org/10.1119/1.1286663

Simple scheme for tunable frequency offset locking of two lasers

Review of Scientific Instruments 70, 242 (1999); https://

doi.org/10.1063/1.1149573

\section{Don't let your writing keep you from getting published!} AIP $\mid$ Author Services 


\title{
Effects of three-body collisions in a two-mode Bose-Einstein condensate
}

\author{
Carlos Sabín, ${ }^{1}$ Pablo Barberis-Blostein, ${ }^{2}$ Cristopher Hernández, ${ }^{3}$ \\ Robert B. Mann, ${ }^{4,5}$ and Ivette Fuentes ${ }^{1}$ \\ ${ }^{1}$ School of Mathematical Sciences, University of Nottingham, \\ Nottingham NG7 2RD, United Kingdom \\ ${ }^{2}$ Instituto de Investigaciones en Matemáticas Applicadas y en Sistemas, Universidad \\ Nacional Autónoma de México, Ciudad de México, Mexico \\ ${ }^{3}$ Instituto de Física, Universidad Nacional Autónoma de México, Ciudad de México, Mexico \\ ${ }^{4}$ Perimeter Institute for Theoretical Physics, 31 Caroline St. N, Waterloo, \\ Ontario N2L 2Y5, Canada \\ ${ }^{5}$ Department of Physics and Astronomy, University of Waterloo, Waterloo, \\ Ontario N2L 3G1, Canada
}

(Received 26 August 2015; accepted 10 November 2015; published online 25 November 2015)

\begin{abstract}
We study the effects of three-body collisions in the physical properties of a two-mode Bose-Einstein condensate. The model introduced here includes two-body and threebody elastic and mode-exchange collisions and can be solved analytically. We will use this fact to show that three-body interactions can produce drastic changes in the probability distribution of the ground state and the dynamics of the relative population. In particular, we find that three-body interactions under certain circumstances may inhibit the collapse of the relative population. (C) 2015 AIP Publishing LLC. [http://dx.doi.org/10.1063/1.4936314]
\end{abstract}

\section{INTRODUCTION}

Most of our understanding of condensed matter is based on models that consider two-body collisions. However there are many situations where three-body or higher order collisions are relevant in the physical properties of such systems. ${ }^{1-3}$ For example, it is known that three-body collisions are important in systems that show exotic quantum phases, such as topological phases ${ }^{2}$ or spin liquids. ${ }^{3}$ Moreover, it is suspected that many-body collisions are important in the coldest phases of Bose-Einstein condensates (BECs), where the dilute regime breaks down. ${ }^{4}$ Microscopic calculations show that polar molecules driven by microwave fields undergo three-body interactions. ${ }^{5}$ The interaction potentials of molecules trapped in an optical lattice give rise to Hubbard models with strong nearest-neighbour two-body and three-body interactions.

In this paper, we find the exact analytic solution of a generalized two-mode Bose-Hubbard model that includes two-body and three-body elastic and mode-exchange collisions. Then we show that three-body collisions can change dramatically the properties of the ground state of a two-mode Bose-Einstein condensate. The effects are also observable in the evolution of the relative population inhibiting, in some cases, quantum collapse. It is well known that three-body collisions are responsible for particle loss in Bose-Einstein condensates, through a process called three-body recombination. ${ }^{6}$ During three-body collisions, the particles recombine to form a molecule that is not trapped by the potential. However it is now possible to inhibit molecular three-body recombination in atomic Bose-Einstein condensates via the application of resonant $2 \pi$ laser pulses. ${ }^{7}$ In such situations, our model becomes of special interest, since it takes into account three-body collisions where particles do not recombine and remain trapped in the potential.

The model we introduce can describe the physics of a double-well Bose-Einstein condensate or a spin-1/2 Bose-Einstein condensate consisting of particles with two internal degrees of freedom trapped in a single well. In the context of the double-well Bose-Einstein condensate, the modeexchange collisions included here are known as generalized nearest neighbour interactions ${ }^{8}$ and give rise to coherent tunneling effects. ${ }^{9,10}$ Recent analysis shows that stronger two-body interactions are 
correlated with two-body coherent tunneling dynamics in which two particles simultaneously tunnel through the barrier. ${ }^{10}$ This effect, also known as second order tunneling, has been observed in the laboratory. ${ }^{9}$ Mode-exchange collisions are called inelastic collisions in the context of spin- $1 / 2$ condensates and occur when cold collisions take place in the presence of light fields. Such is the case of spin- $1 / 2$ condensates where a laser field is used to induce Josephson-type interactions, which produce transitions among the spin degrees of freedom. ${ }^{11}$

\section{MODEL}

We consider a general model of a two-mode Bose-Einstein condensate that includes two-body and three-body collisions given by the Hamiltonian $\mathcal{H}_{3}=H_{1}+H_{2}+H_{3}$, where (taking $\hbar=1$ )

$$
\begin{aligned}
H_{1}= & \lambda_{a \mid a} a^{\dagger} a+\lambda_{b \mid b} b^{\dagger} b+\lambda_{a \mid b}\left(a^{\dagger} b+b^{\dagger} a\right), \\
H_{2}= & \mathcal{U}_{a a \mid a a} a^{\dagger} a^{\dagger} a a+\mathcal{U}_{b b \mid b b} b^{\dagger} b^{\dagger} b b+\mathcal{U}_{a b \mid a b} a^{\dagger} b^{\dagger} a b \\
& +U_{a a \mid a b}\left(a^{\dagger} a^{\dagger} a b+h . c .\right)+U_{b b \mid a b}\left(b^{\dagger} b^{\dagger} a b+h . c .\right) \\
& +U_{a a \mid b b}\left(a^{\dagger} a^{\dagger} b b+h . c .\right) \\
H_{3}= & \mathcal{U}_{a a a \mid a a a} a^{\dagger} a^{\dagger} a^{\dagger} a a a+\mathcal{U}_{b b b \mid b b b} b^{\dagger} b^{\dagger} b^{\dagger} b b b \\
& \mathcal{U}_{a a b \mid a a b} a^{\dagger} a^{\dagger} b^{\dagger} a a b+\mathcal{U}_{a b b \mid a b b} a^{\dagger} b^{\dagger} b^{\dagger} a b b \\
& +U_{a a a \mid a a b}\left(a^{\dagger} a^{\dagger} a^{\dagger} a a b+h . c .\right) \\
& +U_{b b b \mid a b b}\left(b^{\dagger} b^{\dagger} b^{\dagger} a b b+h . c .\right) \\
& +U_{a a a \mid a b b}\left(a^{\dagger} a^{\dagger} a^{\dagger} a b b+h . c .\right) \\
& +U_{b b b \mid a a b}\left(b^{\dagger} b^{\dagger} b^{\dagger} a a b+h . c .\right) \\
& +U_{a a b \mid a b b}\left(a^{\dagger} a^{\dagger} b^{\dagger} a b b+h . c .\right) \\
& +U_{a a a \mid b b b}\left(a^{\dagger} a^{\dagger} a^{\dagger} b b b+\text { h.c. }\right) .
\end{aligned}
$$

The operators $a^{\dagger}, a$ and $b^{\dagger}, b$ are associated with two modes, labeled $\mathrm{A}$ and $\mathrm{B}$, with respective frequencies $\lambda_{a \mid a}$ and $\lambda_{b \mid b}$. These modes correspond either to atoms with two different hyperfine levels ${ }^{12}$ or two spatially separated condensates ${ }^{13,14}$ (see Fig. 1). The Josephson-type interaction, in which one particle is annihilated in one mode and created in the other, has coupling constant $\lambda_{a \mid b}$. This process is induced by applying a magnetic field gradient ${ }^{13}$ or a laser. ${ }^{12}$ The terms in $\mathrm{H}_{2}$, which have four bosonic operators, describe two-particle collisions. The two-body elastic scattering strengths are given by $\mathcal{U}_{a a \mid a a}$ and $\mathcal{U}_{b b \mid b b}$ for same mode collisions and $U_{a b \mid a b}$ when the particles colliding belong to different modes. Mode-exchange collisions have interaction strengths $U_{a a \mid a b}, U_{b b \mid a b}$ when two particles collide and one of them is transformed into the other mode and interaction strength $U_{a a \mid b b}$ when the collision transforms two particles in one mode into the other mode. This process is also known as second order tunneling in the context of a double-well BEC. ${ }^{9,10}$

The Hamiltonian $\mathcal{H}_{2}=H_{1}+H_{2}$ has been studied in detail in Ref. 15. This two-body interaction Hamiltonian coincides with the two-mode Bose-Hubbard model if mode-exchange collisions are neglected, i.e., $U_{a a \mid a b}=U_{b b \mid a b}=U_{a a \mid b b}=0$. However, microscopic calculations show that such

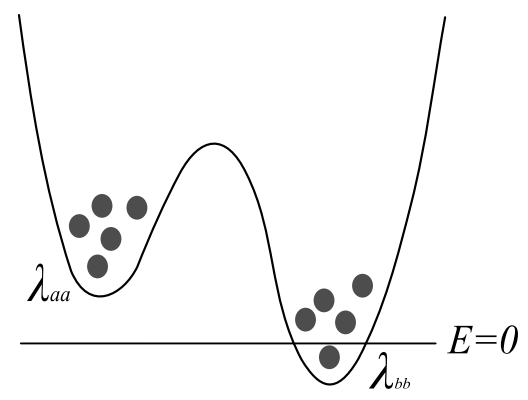

FIG. 1. A Bose-Einstein condensate in an asymmetric double-well potential, characterised by the single well energies $\lambda_{a \mid a}$ and $\lambda_{b \mid b}$. 
interactions, known as inelastic collisions in the context of spin-1/2 Bose-Einstein condensates, should be considered since they occur when particles collide in the presence of a laser field. ${ }^{11}$ Surprisingly, including such collisions allows for an exact analytic solution. ${ }^{15-18}$ Here we include a three-body collision term given by $H_{3}$, where three-body interactions consist of products of six operators (three creation and three annihilation). This term includes all possible three-body collisions where $\mathcal{U}_{a a a \mid a a a}, \mathcal{U}_{b b b \mid b b b}, \mathcal{U}_{a a b \mid a a b}$, and $\mathcal{U}_{a b b \mid a b b}$ correspond to elastic scattering lengths and $\mathcal{U}_{a a a \mid a a b}, \mathcal{U}_{a a a \mid a b b}$, and $\mathcal{U}_{a a a \mid b b b}$ correspond to mode-exchange collisions where one, two, and three particles change mode, respectively.

We have found that the Hamiltonian $\mathcal{H}_{3}$ has six families of exact analytical solutions. In this paper, we present the solution which we consider of greatest physical interest. The other solutions will be presented elsewhere.

We start by considering the double-well potential shown in Fig. 1. Particles undergo two- and three-body collisions and we assume that first, second, and third order tunneling events can occur. In second (third) order tunneling, two (three) tunneling events can occur coherently. Therefore single particles can coherently tunnel two (three) times and two (three) particles can tunnel simultaneously during a collision.

We consider that a particle in well A (or B) has probability amplitude $A_{1} \cos \theta$ (or $-A_{1} \cos \theta$ ) of staying in well A (or B) and probability amplitude $A_{1} \sin \theta$ of tunneling to well B (or A). $A_{1}$ is the first order tunneling strength and $\theta$ is the tunneling phase. Note that the minus sign appears because we chose for simplicity well B to have negative energy corresponding to $\lambda_{b \mid b}=-\lambda_{a \mid a}$. We consider $A_{2}$ and $A_{3}$ to be second and third order tunneling strengths. Therefore, $A_{2} \sin ^{2} \theta$ and $A_{3} \sin ^{2} \theta \cos \theta$, for example, are the second and third order probability amplitudes, respectively, for a single particle in well A to tunnel back and forth.

The coefficients in the single particle Hamiltonian $H_{1}$ are found by considering all possible single particle events including second and third order tunneling. For example,

$$
\begin{aligned}
\lambda_{a \mid a} & =A_{1} \cos \theta+A_{2}\left(\cos ^{2} \theta+\sin ^{2} \theta\right) \\
& +A_{3} \cos \theta\left(\cos ^{2} \theta+\sin ^{2} \theta\right)=A_{2}+\left(A_{3}+A_{1}\right) \cos \theta
\end{aligned}
$$

is the probability amplitude for a single particle in well A to end in well A. The general two-body and three-body scattering lengths $U_{i j \mid l m}$ and $U_{i j k \mid l m n}$ are given by the product of the corresponding second and third order tunneling strengths times the appropriate tunneling phase amplitudes $(\sin \theta$ if the particle tunnels during the collision and $\pm \cos \theta$ if the particle stays). For example, consider a three-body collision during which two particles change state. The total probability amplitude will be

$$
U_{a a a \mid a b b}=3 A_{3} \cos \theta \sin ^{2} \theta .
$$

The factor 3 comes from the fact that there are three possible events that give rise to the same final outcome, according to the different time orderings of the events.

In the case of two-body collisions, we consider that during a collision, two and three tunneling events can occur. So collisions in which two particles in well A end up both in well B are given by

$$
\begin{aligned}
U_{a a \mid b b} & =A_{2} \sin ^{2} \theta \\
& +3 A_{3}\left(\cos \theta \sin ^{2} \theta-\sin ^{2} \theta \cos \theta\right)=A_{2} \sin ^{2} \theta,
\end{aligned}
$$

where again the factor 3 comes from the time ordering. Such considerations give rise to the parameters

$$
\begin{aligned}
\lambda_{a \mid a} & =A_{2}+\left(A_{3}+A_{1}\right) \cos \theta, \\
\lambda_{b \mid b} & =A_{2}-\left(A_{3}+A_{1}\right) \cos \theta, \\
\lambda_{a \mid b} & =\left(A_{1}+A_{3}\right) \sin \theta, \\
\mathcal{U}_{a a \mid a a} & =\left(A_{2} \cos \theta+3 A_{3}\right) \cos \theta, \\
\mathcal{U}_{b b \mid b b} & =\left(A_{2} \cos \theta-3 A_{3}\right) \cos \theta, \\
\mathcal{U}_{a b \mid a b} & =2 A_{2}\left(\sin ^{2} \theta-\cos ^{2} \theta\right), \\
U_{a a \mid a b} & =\left(3 A_{3}+2 A_{2} \cos \theta\right) \sin \theta, \\
U_{b b \mid a b} & =\left(3 A_{3}-2 A_{2} \cos \theta\right) \sin \theta,
\end{aligned}
$$




$$
\begin{aligned}
\mathcal{U}_{a a \mid b b} & =A_{2} \sin ^{2} \theta, \\
\mathcal{U}_{b b b \mid b b b} & =-\mathcal{U}_{\text {aaa } \mid a a a}=-A_{3} \cos ^{3} \theta, \\
\mathcal{U}_{a b b \mid a b b} & =-\mathcal{U}_{a a b \mid a a b}=-A_{3}\left(2 \cos \theta \sin ^{2} \theta-\cos ^{3} \theta\right), \\
U_{a a a \mid a a b} & =U_{b b b \mid a b b}=3 A_{3} \cos ^{2} \theta \sin \theta, \\
U_{a a a \mid a b b} & =3 A_{3} \cos \theta \sin ^{2} \theta, \\
U_{b b b \mid a a b} & =-3 A_{3} \cos \theta \sin ^{2} \theta, \\
U_{a a b \mid a b b} & =3 A_{3}\left(\sin ^{3} \theta-\cos ^{2} \theta \sin \theta\right), \\
\mathcal{U}_{a a a \mid b b b} & =A_{3} \sin ^{3} \theta .
\end{aligned}
$$

At this point, it is important to illustrate the connection of this model with a physical model of a BEC. Consider the many-body energy functional for bosonic particles of mass $m$ trapped in a potential $V(\mathbf{r})$ undergoing two-body and three-body collisions,

$$
\begin{aligned}
H & =H_{0}+H_{I 2}+H_{I 3}, \\
H_{0} & =\int d \mathbf{r}\left(-\frac{\hbar^{2}}{2 m} \hat{\Psi}^{\dagger} \nabla^{2} \hat{\Psi}+\hat{\Psi}^{\dagger} V(\mathbf{r}) \hat{\Psi}\right) \\
& =\int d \mathbf{r} \hat{\Psi}^{\dagger} H_{t} \hat{\Psi}, \\
H_{I 2} & =\frac{g_{2}}{2} \int d \mathbf{r} \hat{\Psi}^{\dagger} \hat{\Psi}^{\dagger} \hat{\Psi} \hat{\Psi}, \\
H_{I 3} & =\frac{g_{3}}{2} \int d \mathbf{r} \hat{\Psi}^{\dagger} \hat{\Psi}^{\dagger} \hat{\Psi} \hat{\varphi} \hat{\Psi} \hat{\Psi} \hat{\Psi},
\end{aligned}
$$

where $g_{2}, g_{3}$ are two-body and three-body coupling strengths, respectively, and $H_{t}$ is the Hamiltonian of the trap. The wavefunction $\hat{\Psi}$ can be expanded in terms of a certain set of functions $\phi_{i}$ and their corresponding annihilation operators $\hat{c}_{i}$ as

$$
\hat{\Psi}=\sum_{i} \phi_{i} \hat{c}_{i}
$$

We employ the standard two-mode approximation

$$
\hat{\Psi}=\phi_{1} \hat{c}_{1}+\phi_{2} \hat{c}_{2}
$$

and then the rotation

$$
\begin{aligned}
\phi_{1} & =\cos (\theta / 2) \phi_{a}-\sin (\theta / 2) \phi_{b}, \\
\phi_{2} & =\cos (\theta / 2) \phi_{b}+\sin (\theta / 2) \phi_{a},
\end{aligned}
$$

where $\phi_{a}, \phi_{b}$ are nearly normalized modes ${ }^{19}$ with $\int d r \phi_{a} \phi_{a}=1+\epsilon, \int d r \phi_{b} \phi_{b}=1-\epsilon$, where the amplitude of transition between them

$$
\epsilon=\int d r \phi_{a} H_{t} \phi_{b}
$$

is assumed to be very small. We obtain the Hamiltonian $\mathcal{H}_{3}$ described above with

$$
\begin{array}{r}
A_{1}=\frac{1}{2}\left(E_{1}-E_{2}\right), A_{2}=\frac{1}{2} U_{2}, U_{2}=U_{11}=U_{22}, A_{3}=\frac{1}{2} U_{3}, U_{3}=U_{111}=U_{222}, \\
E_{1}=\int d r \phi_{1} H_{t} \phi_{1}, U_{11}=\int d r \phi_{1}^{4}, U_{111}=\int d r \phi_{1}^{6}, \\
E_{2}=\int d r \phi_{2} H_{t} \phi_{2}, U_{22}=\int d r \phi_{2}^{6}, U_{222}=\int d r \phi_{2}^{6}
\end{array}
$$

plus several terms of order $\epsilon, \epsilon^{2}$, and $\epsilon^{3}$ which can be treated perturbatively ${ }^{17,18}$ as long as $\epsilon \ll A_{1} \theta$. We will discuss the experimental validity of this approximation in Sec. IV. 


\section{RESULTS}

Surprisingly, the Hamiltonian $\mathcal{H}_{3}=H_{1}+H_{2}+H_{3}$ has an exact analytic solution for this set of parameters. The analytic expression of its eigenstates is

$$
\left|E_{m}\right\rangle=e^{\frac{-\theta}{2}\left(a^{\dagger} b-a b^{\dagger}\right)}|J, m\rangle,
$$

where $|J, m\rangle$ are the Fock states for $2 J=N . N$ is the total number of particles, given by the operator

$$
\hat{N}=n_{a}+n_{b}=a^{\dagger} a+b^{\dagger} b,
$$

and $m$ is the eigenvalue of the relative population operator,

$$
\hat{m}=\left(a^{\dagger} a-b^{\dagger} b\right) / 2 \text {. }
$$

Since the number of particles in the system $N$ is constant, $m$ is restricted to values $m=-J, \ldots, J$. The unitary operator $e^{\frac{\theta}{2}\left(a^{\dagger} b-a b^{\dagger}\right)}$ is known as the two-mode displacement operator with real displacement parameter $\theta$.

Interestingly, the eigenstate for $m=-J$ corresponds to a coherent state, which gives an appropriate description of several physical aspects of the two-mode Bose-Einstein condensate. ${ }^{20}$ It is easy to verify that $e^{\frac{-\theta}{2}\left(a^{\dagger} b-a b^{\dagger}\right)}|J, m\rangle$ are the eigenstates of Hamiltonian (1). One must simply apply the two-mode displacement operator to the Hamiltonian,

$$
H_{0}=A_{1}\left(a^{\dagger} a-b^{\dagger} b\right)+A_{2}\left(a^{\dagger} a-b^{\dagger} b\right)^{2}+A_{3}\left(a^{\dagger} a-b^{\dagger} b\right)^{3},
$$

which is diagonal in the Fock basis. The result of this transformation is the Hamiltonian $\mathcal{H}_{3}$ with the coefficients shown in (5), except for an energy shift. For this reason, both Hamiltonians have the same energy spectrum and their eigenvectors are related by the displacement operator. The ground state of the system is $\left|E_{g}\right\rangle=e^{\frac{-\theta}{2}\left(a^{\dagger} b-a b^{\dagger}\right)}\left|J, m_{0}\right\rangle$, where $m_{0}$ is the integer that minimizes the energy $E_{m}=A_{1} m+A_{2} m^{2}+A_{3} m^{3}$. This number can be determined with the expressions,

$$
\begin{array}{rlrl}
m_{0}^{ \pm} & =\frac{A_{2}}{3 A_{3}}\left(-1 \pm \sqrt{1-\frac{3 A_{1} A_{3}}{A_{2}^{2}}}\right), A_{3} \neq 0, \\
m_{0} & =-\frac{A_{1}}{2 A_{2}}, & A_{3}=0 .
\end{array}
$$

If $3 A_{1} A_{3} / A_{2}^{2}>1, m_{0}$ is a complex number and the energy has no local minimum. Therefore, the minimum energy will correspond to the extreme point $m_{e x}=-N A_{3} /\left|A_{3}\right|$. On the other hand, if $3 A_{1} A_{3} / A_{2}^{2}<1$ then the minimum, which is given by Eq. (16), is $m_{0}^{+}$for $A_{3}>0$ and $m_{0}^{-}$when $A_{3}<0$. However, if the size of the system is big enough, then the cubic part of the energy prevails for $|m| \gg 0$. Under these circumstances, the energy of the extreme point $E_{m_{e x}}$ is smaller than the local minimum $E_{m_{0}^{ \pm}}$.

A quantity of interest is the probability distribution of the relative population for the ground state, which is given by

$$
P=\left|\left\langle N, m \mid \psi_{0}\right\rangle\right|^{2}=\left|d_{m, m_{0}}^{N}\right|^{2},
$$

where

$$
\begin{aligned}
d_{m, m_{0}}^{N}=\sum_{k}(-1)^{k-m_{0}+m} \frac{\sqrt{\left(N+m_{0}\right) !\left(N-m_{0}\right) !(N+m) !(N-m) !}}{\left(N+m_{0}-k\right) ! k !(N-k-m) !\left(k-m_{0}+m\right) !} \\
\\
\quad \times\left(\cos \frac{\theta}{2}\right)^{2 N-2 k+m_{0}-m}\left(\sin \frac{\theta}{2}\right)^{2 k-m_{0}+m}
\end{aligned}
$$

are the Wigner rotation matrix elements. ${ }^{21}$ Note that the sum must be done for the values of $k$ such that none of the arguments of the factorials in the denominator are negative. Different ground states parametrized by $m_{0}$ are obtained by changing the rate $A_{1} A_{3} / A_{2}^{2}$. We plot in Fig. 2 an example for $N=100$ particles with $A_{3}=0$ (i.e., assuming that there are no third order tunneling and three-body collisions) and $m_{0}=A_{1} / 2 A_{2}=-50$. Such a state has a multi-peak distribution. However, if $A_{3}=0.0035$, we get a single peak distribution corresponding to $m_{0}=-100$, i.e., a coherent state. Each figure has an inset 

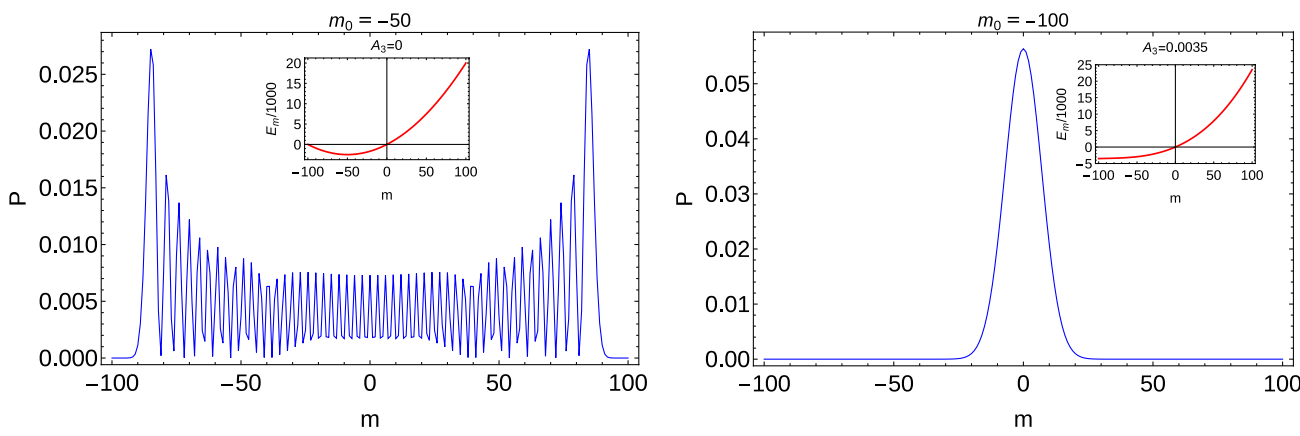

FIG. 2. Probability distribution $\mathrm{P}$ of the relative population for the ground state with $\mathrm{N}=100, A_{1} / A_{2}=100$ and (left) $A_{3}=0$, (right) $A_{3}=0.0035$. The ground state distribution changes from a multi-peak to a single peak distribution if three-body collisions are included. The insets show the change in the spectrum.

with a plot of the corresponding spectrum, where we can see that three body processes generate a shift of the minimum. This shows how three-body collisions and third order tunneling drastically change the structure of the ground state of the system. Moreover, the energy gap between the ground and first excited states is larger in Fig. 2(b). This is a typical feature of the self-trapping regime ${ }^{22}$ which suggests that three-body terms tend to favor localization. We will confirm this insight below.

We now analyze the effects of three-body collisions on the evolution of the average relative population $\langle m\rangle=\left\langle a^{\dagger} a-b^{\dagger} b\right\rangle$, for an initial condition $|\psi(t=0)\rangle$. The evolution of the relative population is given by

$$
\begin{aligned}
\langle m\rangle & =\cos \theta \sum_{-N}^{N} m\left|C_{m}\right|^{2} \\
& -\sin \theta \sum_{-N+1}^{N} C_{m} C_{m-1} \sqrt{N(N+1)-m(m-1)} L_{m}, \\
L_{m} & =\cos \left[\left(E_{m-1}-E_{m}\right) t\right],
\end{aligned}
$$

where the coefficients $C_{m}$ are defined by

$$
|\psi(t=0)\rangle=\sum_{m=-N}^{N} C_{m} e^{\frac{-\theta}{2}\left(a^{\dagger} b-a b^{\dagger}\right)}|N, m\rangle .
$$

In Fig. 3, we plot Equation (19) for $N=100, A_{1}=100, A_{2}=1$ and the initial condition $|\psi(t=0)\rangle=$ $|N, N\rangle$, i.e., all the particles start in a single well. We can see that the relative population shows collapses and revivals for two body collisions. We can also observe that a small rate of three-body collisions $\left(A_{3}=1 / 100\right)$ has a noticeable effect on the behaviour of the time evolution of the system, and in fact, it tends to break down the perfect collapse-revival cycles of the relative population. These cycles are characteristic of a delocalized dynamics, while the small-amplitude oscillations of the population imbalance in Fig. 3(b) point to a self-trapped dynamics. This confirms that the three-body terms tend to favor localization in this model.

\section{DISCUSSION AND CONCLUSIONS}

At this point, let us discuss the experimental relevance of our results. In the case of a doublewell potential, the modes $\mathrm{a}$ and $\mathrm{b}$ are quasilocalised modes in wells $\mathrm{A}$ and $\mathrm{B}$ and $A_{1}$ corresponds to the asymmetry between the two wells. Thus, $\epsilon$ and $A_{1}$ can be experimentally tuned by changing the distance and the energy offset between the wells, respectively. Indeed, as discussed in Ref. 17, the condition $\epsilon \ll A_{1} \theta$ holds in a wide variety of experiments with double-well BECs. ${ }^{23-25}$ More specifically, the linear tunnelling rate $U_{1 \mid 2}=\int d r \phi_{1} H_{t} \phi_{2} \simeq A_{1} \theta+\epsilon$ takes experimental values ranging from $5 \cdot 10^{-4} \mathrm{~Hz} \mathrm{~h}^{24}$ to $2 \mathrm{~Hz} \mathrm{~h},{ }^{23}$ while the energy offset between the wells can be as high as $A_{1}=530 \mathrm{~Hz} \mathrm{h.}{ }^{25}$ Even if the wells are intended to be perfectly symmetric, the 



FIG. 3. Evolution of the relative population $\langle m\rangle$ for $\mathrm{N}=100$ particles with $A_{1}=100, A_{2}=1$, and $A_{3}$ specified in each figure. The initial state corresponds to $|\psi(t=0)\rangle=|N, N\rangle$. We observe that the presence of three-body collisions changes the dynamics of the system and in fact tends to break down perfect quantum collapse-revival cycles. We show that this phenomenon occurs in a continuous fashion with respect to the parameter $A_{3}$.

uncertainty in the trap depth leads us to assume a minimum trap asymmetry of $A_{1} \simeq 20 \mathrm{~Hz} \mathrm{h.}{ }^{23}$ Regarding the other parameters, $A_{2}$ and $A_{3}$ represent the two-body and three-contributions to the potential energy. It has been estimated the latter represent a few percent of the former. For instance, in $\mathrm{He},{ }^{1} A_{3}$ is around $2 \%$ of the total potential energy. All these values are in line with the plots in Sec. III. Note also that the three-body terms represent the leading-order corrections to the standard two-body interaction. It is reasonable to assume that four-body and higher order terms will be small with respect to the three-body ones and overall negligible. We can conclude that our model is suitable to describe a wide variety of experiments involving double-well BECs. It would be interesting to explore as well the microscopic derivation of a spin-1/2 BEC in order to determine the connection with our model.

In summary, we introduced a model of two-mode Bose-Einstein condensate which includes not only two-body but also three-body interactions. We find an analytic solution and provide the full spectrum of eigenvalues and the corresponding eigenvectors. This allows us to analyse the role of three-body interactions in physical quantities of interest, such as the probability distribution of the relative population or the time evolution of its expectation value. We find that three-body collisions have non-trivial effects, such as significant changes in the probability distribution of the ground state or the inhibition of collapses in the evolution of the relative population of the modes. Our work provides insights into the effects of higher order collisions in the physics of a two component Bose-Einstein condensate. Following the formalism employed in this paper, higher-order collisions can also be included in the model. ${ }^{16}$

\section{ACKNOWLEDGMENTS}

I.F. and C.S. acknowledge funding from EPSRC (CAF Grant No. EP/G00496X/2 to I.F.). R.B.M. was supported by the Natural Sciences and Engineering Research Council of Canada. 
${ }^{1}$ R. D. Murphy and J. A. Barker, "Three-body interactions in liquid and solid helium,” Phys. Rev. A 3, 1037 (1971).

${ }^{2}$ N. R. Cooper, "Exact ground states of rotating Bose gases close to a Feshbach resonance," Phys. Rev. Lett. 92, 220405 (2004).

${ }^{3}$ L. Balents, M. P. A. Fisher, and S. M. Girvin, "Fractionalization in an easy-axis Kagome antiferromagnet," Phys. Rev. B 65, 224412 (2002).

${ }^{4}$ M. Inguscio, S. Stringari, and C. Wieman, Bose-Einstein Condensation in Atomic Gases (IOS Press, 1999)

${ }^{5}$ H. P. Büchler, A. Micheli, and P. Zoller, "Three-body interactions with cold polar molecules," Nat. Phys. 3, 726 (2007).

${ }^{6}$ T. B. Laburthe et al., "Observation of reduced three-body recombination in a correlated 1D degenerate Bose gas," Phys. Rev. Lett. 92, 190401 (2004).

${ }^{7}$ C. P. Search, W. Zhang, and P. Meystre, "Inhibiting three-body recombination in atomic Bose-Einstein condensates," Phys. Rev. Lett. 92, 140401 (2004).

${ }^{8}$ H. Heiselberg, "Extended Bose-Hubbard model with incompressible states at fractional numbers," Phys. Rev. A 73, 013628 (2006).

${ }^{9}$ S. Fölling et al., "Direct observation of second-order atom tunnelling," Nature 448, 1029 (2007).

${ }^{10}$ J.-Q. Liang, J.-L. Liu, W.-D. Li, and Z.-J. Li, "Atom-pair tunneling and quantum phase transition in the strong-interaction regime," Phys. Rev. A 79, 033617 (2009).

${ }^{11} \mathrm{P}$. S. Julienne, "Estimating bounds on collisional relaxation rates of spin-polarized $87 \mathrm{Rb}$ atoms at ultracold temperatures," J. Res. Natl. Inst. Stand. Technol. 101, 487 (1996).

${ }^{12}$ C. J. Myatt, E. A. Burt, R. W. Ghrist, E. A. Cornell, and C. E. Wieman, "Production of two overlapping Bose-Einstein condensates by sympathetic cooling," Phys. Rev. Lett. 78, 586 (1997).

${ }^{13}$ J. Stenger et al., "Spin domains in ground-state Bose-Einstein condensates," Nature (London) 396, 345 (1998).

${ }^{14}$ H. J. Miesner et al., "Observation of metastable states in spinor Bose-Einstein condensates," Phys. Rev. Lett. 82, 2228 (1999).

${ }^{15}$ P. Barberis-Blostein and I. Fuentes-Schuller, "Mode-exchange collisions in an exactly solvable two-mode Bose-Einstein condensate," Phys. Rev. A 78, 013641 (2008).

${ }^{16}$ P. Barberis-Blostein and I. Fuentes-Schuller, "A family of many-body models which are exactly solvable analytically," J. Phys. A: Math. Theor. 40, F601 (2007).

${ }^{17}$ C. Sabín, P. Barberis-Blostein, and I. Fuentes, “Analytical solution of a double-well Bose-Einstein Condensate," e-print arXiv:1406.4984 (2014).

${ }^{18}$ R. B. Mann, M. B. Young, and I. Fuentes-Schuller, "A perturbative approach to inelastic collisions in a Bose-Einstein condensate," J. Phys. B: At., Mol. Opt. Phys. 44, 085031 (2011).

${ }^{19}$ G. J. Milburn, J. Corney, E. M. Wright, and D. F. Walls, "Quantum dynamics of an atomic Bose-Einstein condensate in a double-well potential,” Phys. Rev. A 55, 4318 (1997).

${ }^{20}$ D. Gordon and C. M. Savage, "Creating macroscopic quantum superpositions with Bose-Einstein condensates," Phys. Rev. A 59, 4623 (1999).

${ }^{21}$ J. J. Sakurai, Modern Quantum Mechanics (Addison-Wesley Publishing Company, 1994).

${ }^{22}$ D. Rubeni, A. Foerster, E. Mattei, and I. Roditi, "Quantum phase transitions in Bose-Einstein condensates from a Bethe ansatz perspective,” Nucl. Phys. B 856, 698 (2012).

${ }^{23}$ M. Albiez et al., "Direct observation of tunneling and nonlinear self-trapping in a single Bosonic Josephson Junction," Phys Rev. Lett. 95, 010402 (2005).

${ }^{24}$ Y. Shin et al., "Atom interferometry with Bose-Einstein condensates in a double-well potential," Phys. Rev. Lett. 92, 050405 (2004).

${ }^{25}$ M. Saba et al., "Light scattering to determine the relative phase of two Bose-Einstein condensates," Science 307, 1945 (2005). 